\title{
IdeAs
}

Idées d'Amériques

$11 \mid 2018$

Modernités dans les Amériques : des avant-gardes à aujourd'hui

\section{Entre transformations et résistances : enseignants et étudiants pendant le « mai argentin »}

Transformations and Resistances: Teachers and Students during May of 68 in Argentina

Entre transformaciones y resistencias : docentes y estudiantes durante el "Mayo argentino"

\section{Antonio Ramos Ramírez}

\section{OpenEdition}

\section{Journals}

Édition électronique

URL : https://journals.openedition.org/ideas/2312

DOI : $10.4000 /$ ideas.2312

ISSN : 1950-5701

Éditeur

Institut des Amériques

Référence électronique

Antonio Ramos Ramírez, «Entre transformations et résistances : enseignants et étudiants pendant le " mai argentin » », IdeAs [En ligne], 11 | 2018, mis en ligne le 18 juin 2018, consulté le 20 octobre 2022 URL : http://journals.openedition.org/ideas/2312 ; DOI : https://doi.org/10.4000/ideas.2312

Ce document a été généré automatiquement le 20 octobre 2022.

\section{(c) (i) (9)}

Creative Commons - Attribution - Pas d'Utilisation Commerciale - Pas de Modification 4.0 International - CC BY-NC-ND 4.0

https://creativecommons.org/licenses/by-nc-nd/4.0/ 


\title{
Entre transformations et
} résistances : enseignants et étudiants pendant le « mai argentin »

\author{
Transformations and Resistances: Teachers and Students during May of 68 in \\ Argentina
}

Entre transformaciones y resistencias : docentes y estudiantes durante el "Mayo argentino"

Antonio Ramos Ramírez

1 Contrairement à d'autres régions du monde, l'année 1968 ne marqua pas en Argentine un point de clivage entre des structures sociopolitiques en décomposition et l'apparition d'une nouvelle génération de militants. La réplique la plus nette du "moment 68" en Argentine eut lieu en mai 1969, au cours des journées de lutte du Cordobazo. Le 29 et 30 mai, les étudiants et ouvriers de Córdoba expriment leur action collective à travers la grève politique de masse contre les forces répressives ${ }^{1}$. Mais, quand il s'agit d'interpréter le «mai argentin » il convient de le faire à travers le prisme de l'instabilité institutionnelle qui jalonne l'histoire politique du pays et des rapports dialectiques qui y surgissent, raison pour laquelle il est pertinent de commencer notre analyse quelques années avant.

2 En juin 1966, le général Juan Carlos Onganía instaure une dictature auto-proclamée Revolución Argentina qui avait pour objectif principal l'installation d'un régime corporatiste qui permettrait la transformation des structures productives et la proscription, voire l'élimination, des acteurs sociaux contraires à ce modèle bureaucratique-autoritaire ${ }^{2}$. L'Onganiato était, essentiellement, un projet enraciné dans les valeurs propres au nationalisme militariste, l'anticommunisme viscéral et le catholicisme réactionnaire qui cherchait à démanteler les mécanismes - survivants du régime antérieur - de transfert des revenus vers les travailleurs. 
3 La cartographie du conflit social de cette période se déplaça vers l'intérieur du pays, suivant une logique centrifuge qui brisa les imaginaires centralistes des pouvoirs siégeant à Buenos Aires. Le déroulement des événements du «mai argentin» le confirme: Corrientes, Rosario, Salta, Tucumán, Córdoba, Catamarca, Jujuy et Mendoza, avec des épisodes de contestation et d'insurrection populaire entre 1969-1972, démontrent qu'en Argentine les conflits sociaux du 68 long s'intensifiaient au-delà de la capitale et de sa province.

4 L'arrivée au pouvoir d'Onganía signifie un point de non-retour générationnel: les pratiques répressives de l'État, l'exil de l'ancien dirigeant Perón, expulsé depuis plus de dix ans, et la référence cubaine du triomphe de la voie armée expliquent l'irruption sur la scène d'une génération de jeunes (ouvriers, lycéens et étudiants universitaires) en voie de radicalisation politique. Cette génération - qu'on appellera ultérieurement les setentistas - sera l'acteur principal des épisodes de lutte du "mai argentin", où l'intersection entre le monde éducatif et les transformations politiques s'avère instructif.

\section{Politiques de la dictature et confrontation croissante}

La mise en œuvre des réformes économiques, conçues par les ministres Jorge Salimei et son successeur Adalbert Krieger Vasena, passe dans un premier temps par le démantèlement d'un appareil productif traditionnel fortement syndicalisé. La restructuration industrielle cible de manière particulièrement intense la province de Tucumán, dans le nord-ouest du pays. Le complexe agro-industriel des ingenios sucriers fut l'objet du décret 16.926 du 22 août 1966, signé à peine un mois après la prise du pouvoir par les militaires. La fermeture progressive de onze usines, entre 1966-1968, est à l'origine d'une forte mobilisation populaire, ouvrière mais aussi étudiante, inédite dans une région qui était considérée comme la poudrière de la république.

La présidence d'Onganía, avec plus d'élan que les gouvernements militaires précédents, n'hésitait pas à réprimer les protestations avec l'utilisation décomplexée des ressorts de la violence institutionnelle. Parmi les travailleurs sucriers, la première victime fut Hilda Guerrero de Molina ${ }^{3}$, épouse d'un ouvrier licencié de l'ingenio Santa Lucía. Le 12 janvier 1967, lors d'une mobilisation arrivant à l'ingenio Bella Vista, Guerrero de Molina tomba sous les balles d'un officier de police lorsque, éloignée du centre de l'affrontement, elle traversait la rue pour se protéger des gaz lacrymogènes ${ }^{4}$. L'assassinat de Guerrero de Molina est devenu un symbole de la lutte ouvrière de Tucumán, ce qui explique l'épisode de la pueblada (insurrection locale) de l'ingenio Bella Vista en janvier 1967 ; sa mémoire sera d'ailleurs immédiatement revendiquée par la Confédération Générale du Travail en tant que martyre des travailleurs argentins ${ }^{5}$.

Cependant, la répression du mouvement étudiant avait commencé quelques mois avant. La noche de los bastones largos est le premier acte de la longue histoire de la résistance aux transformations et réformes que le gouvernement d'Onganía essaiera de mettre en place dans le champ éducatif. La suppression de l'autonomie universitaire était, aux yeux de la dictature, une opération stratégique pour chasser l'ennemi interne imaginé par la Doctrine de sécurité nationale. Contestant cette décision, cinq facultés de l'Université de Buenos Aires étaient occupées par des étudiants et des enseignants lorsqu'ils furent violemment délogés dans la nuit du 29 juillet 1966. L'activité politique 
des universitaires ne fera qu'augmenter par la suite, alors que la dictature voulait affirmer que "l'autorité est au-dessus de la science ${ }^{6}$.

8 Le climat d'opposition au sein des universités était croissant et les assemblées et les mobilisations contestataires de tout type s'étaient étendues partout dans le pays. Dans ce contexte d'agitation généralisée, Santiago Pampillón, étudiant et ouvrier originaire de Mendoza, est mort à Córdoba le 12 septembre 1966, après avoir reçu, cinq jours plutôt, trois balles dans la tête tirées à bout portant par un policier en uniforme.

9 Les secteurs populaires en lutte étaient un espace de convergence entre le monde ouvrier et le monde éducatif. Mais si jusqu'ici on a évoqué certains des événements marquants de la lutte ouvrière pendant le «mai argentin », on ne doit pas oublier que la dictature d'Onganía n'a pas épargné l'éducation de sa volonté de transformation réactionnaire, et que les travailleurs de l'éducation l'ont fermement contestée.

\section{Les travailleurs de l'éducation et le « mai argentin »} conquêtes de l'État libéral du XIX ${ }^{\mathrm{e}}$ siècle, garant d'un système éducatif public, obligatoire, gratuit et laïc. Malgré toutes ses limites, la loi 1.420 était interprétée, aussi bien par les travailleurs de l'éducation que par les étudiants engagés, comme le cadre juridique qui permettait de faire de l'Éducation nationale un dispositif de mobilité sociale ascendante. L'Onganiato, par contre, considérait que la loi était un obstacle aux mesures de rationalisation qui devaient dépasser le modèle économique du pays et atteindre aussi le système éducatif ${ }^{7}$.

11 Le projet de réforme de la loi éducative fut présenté par le ministre José Mariano Astigueta, un avocat catholique qui avait déjà occupé le ministère sous la présidence de José María Guido en 1963. Dans le projet de loi de 1968, on trouve déjà des similitudes avec le projet éducatif du Proceso de reorganización - la dictature militaire de 1976-1983 -, plus précisément, en ce qui concerne la conception de l'éducation comme un mécanisme de transmission des valeurs chrétiennes traditionnelles à la culture argentine. Parmi d'autres changements structuraux, le projet de loi visait à réduire le nombre d'années obligatoires dans l'enseignement primaire et l'introduction d'un cycle intermédiaire plus adapté aux besoins du marché de travail. la résistance aux réformes éducatives des niveaux inférieurs d'enseignement fut principalement incarnée par le syndicalisme combatif des enseignants. À ce moment-là, la CGERA (Confédération générale de l'Éducation de la République argentine) fit preuve de sa force organisationnelle, impulsa les actions collectives contre le gouvernement et adopta une position politique claire d'affrontement de la dictature. La politisation des travailleurs de l'éducation était impulsée par les conditions de travail mais aussi par les conditions matérielles de plus en plus précaires auxquelles leurs étudiants étaient soumis.

13 À la suite du Cordobazo, la CGERA décida de se réunir à Cordoba pour préparer l'Assemblée nationale de l'Éducation, afin d'élaborer un projet de loi fédérale d'éducation représentatif des intérêts du collectif. Ainsi, au lendemain du Cordobazo, le secrétariat de CGERA demandait au gouvernement: "La restitution de l'autonomie universitaire et le co-gouvernement étudiant; la dérogation de la loi universitaire ; 
l'élimination des services d'espionnage et d'information dans les universités, et la fin de la privatisation et commercialisation de l'enseignement $\|^{8}$.

Au-delà des manifestations, grèves et négociations menées par le syndicalisme enseignant, l'enjeu de la réforme éducative rassembla la solidarité des syndicats les plus combatifs dont l'exemple le plus évident reste la position prise par la CGTA (Confédération générale du travail des Argentins). La publication périodique de cette fraction combative du mouvement ouvrier dénonçait les salaires insuffisants des enseignants, les répercussions négatives de la décentralisation de l'enseignement primaire et la destruction du patrimoine démocratique ${ }^{9}$.

\section{Considérations finales}

Pour les enseignants et les étudiants argentins, mais on pourrait dire pour la classe ouvrière plus généralement, 1968 est, plus qu'une explosion ponctuelle de colère collective, l'espace temporel d'accélération du conflit social. En décloisonnant les limites chronologiques, le «mai argentin» représente un épisode particulièrement intense de la confrontation entre les secteurs populaires et les élites qui soutenaient le projet de la dictature.

(uttes cumulées au cours du « mai argentin » sont d'une pertinence historique indéniable: l'unité d'action collective entre enseignants, étudiants et ouvriers sert à expliquer le cours des événements des années 70. Cinquante ans après les événements, il est peut être important de rappeler que l'histoire d'un 1968 long en Argentine est aussi celle d'une génération qui paria sur son présent pour construire le futur.

\section{BIBLIOGRAPHIE}

Balvé, Beba C. y Balvé, Beatriz S., El '69 : Huelga política de masas. Rosariazo-Cordobazo-Rosariazo, Buenos Aires, Razón y Revolución, 2005.

Centanni, Antonela, " Autoritarismo y educación en los años “60. El fracaso de una reforma », XVII Jornadas Argentinas de Historia de la Educación, 2012, http://www.ceicargentina.com.ar/ pdfs/autoritarismo-y-educacion-centanni.pdf, page consultée le 4 mai 2018.

De Luca, Roma et Álvarez Prieto, Natalia, « Un análisis comparativo de las reformas educativas orgánicas de los años sesenta y noventa en Argentina. ¿Transformación o consolidación de tendencias ? », Cadernos de História da Educaçao, vol. 15, № 1, 2016, p. 378-397, http://dx.doi.org/ 10.14393/che-v15n1-2016-15, page consultée le 4 mai 2018.

Lorca, Javier, « Pegaban bien, pegaban con ganas », Página 12, https://www.pagina12.com.ar/ diario/especiales/subnotas/70668-22959-2006-07-29.html, page consultée le 4 mai 2018.

Molina, Juan Andrés, « Las voces del pueblo : testimonio de Juan Molina, hijo de Hilda Guerrero de Molina, asesinada por la policía tucumana », San Miguel de Tucumán, octubre 1968, http:// 
icaadocs.mfah.org/icaadocs/ELARCHIVO/RegistroCompleto/tabid/99/doc/758550, page consultée le 5 mai 2018.

Nassif, Silvia, « Resistencia obrera y popular en Tucumán en los inicios de la dictadura de Onganía : asesinato de Hilda Guerrero de Molina y pueblada en Bella Vista », Trabajo y Sociedad, no 27, 2017, p. 195-221, http://www.unse.edu.ar/trabajoysociedad/ 29\%20NASSIF\%20SILVIA\%20\%20FOTIA.pdf, page consultée le 4 mai 2018.

O'Donnell, Guillermo, El Estado burocrático-autoritario : triunfos, derrotas y crisis. Buenos Aires, Editorial de Belgrano, 1982.

\section{NOTES}

1. Balvé, Beba C. y Balvé, Beatriz S., El ‘69 : Huelga política de masas. Rosariazo-CordobazoRosariazo, Buenos Aires, Razón y Revolución, 2005.

2. O'Donnell, Guillermo, El Estado burocrático-autoritario: triunfos, derrotas y crisis. Buenos Aires, Editorial de Belgrano, 1982.

3. Le récit du fils de Hilda Guerrero est issu d'un entretien réalisé par des artistes participant au collectif d'avant-garde artistique Tucumán arde. Comme le signale Ana Longoni, des copies de cette publication furent diffusées aux assistants de l'exposition à Rosario et Buenos Aires.

Molina, Juan Andrés « Las voces del pueblo : testimonio de Juan Molina, hijo de Hilda Guerrero de Molina, asesinada por la policía tucumana », San Miguel de Tucumán, octobre 1968.

4. « Tucumán : la espina en la garganta », Primera plana, № 212, 17-23/01/1967, p. 12.

5. Nassif, Silvia, «Resistencia obrera y popular en Tucumán en los inicios de la dictadura de Onganía : asesinato de Hilda Guerrero de Molina y pueblada en Bella Vista », Trabajo y Sociedad, no 27, 2017, p. 195-221.

6. Lorca, Javier « Pegaban bien, pegaban con ganas », page 12.

7. De Luca, Roma et Álvarez Prieto, Natalia, "Un análisis comparativo de las reformas educativas orgánicas de los años sesenta y noventa en Argentina. ¿Transformación o consolidación de tendencias ?", Cadernos de História da Educaçao, vol. 15, № 1, 2016, p. 378-397.

8. La Gaceta, San Miguel de Tucumán, $01 / 06 / 1969$, p. 13. Nous traduisons.

9. Centanni, Antonela, « Autoritarismo y educación en los años '60. El fracaso de una reforma », XVII Jornadas Argentinas de Historia de la Educación, 2012.

\section{AUTEUR}

\section{ANTONIO RAMOS RAMÍREZ}

Chercheur au Centre d'Histoire Culturelle des Sociétés Contemporaines de l'Université de Versailles-Saint-Quentin-en-Yvelines. 